\title{
Case report: histopathology and molecular pathology analysis on enteric tissue of a COVID-19 patient
}

Yanling Feng ${ }^{{ }^{*+}}$, Dong Zeng ${ }^{1+}$, Lvyin $\mathrm{Hu}^{2+}$, Yuexiang Yang ${ }^{1}$, Shu Song ${ }^{1}$, Yuhan Shi ${ }^{1}$, Jingjing X ${ }^{1}$, Wenjuan Guo ${ }^{1}$, Yun Ling ${ }^{3}$, Tangkai $\mathrm{Qi}^{3}$, Qingguo $\mathrm{Wu}^{4}$, Feng $\mathrm{Li}^{4}$, Jilin Cheng ${ }^{5}$ and Hongzhou $\mathrm{Lu}^{3^{*}}$

\begin{abstract}
Aims: Patients with COVID-19 can also have enteric symptoms. Here we analyzed the histopathology of intestinal detachment tissue from a patient with COVID-19.

Methods: The enteric tissue was examined by hematoxylin \& eosin stain, PAS (Periodic acid-Schiff) staining, Gram staining, Ziehl-Neelsen stain and Grocott's Methenamine Silver (GMS) Stain. The distribution of CD3, CD4, CK20 and CD68, cytomegalovirus (CMV) and Herpes Simplex Virus (HSV) antigen were determined by immunohistochemistry. In situ hybridization (ISH) of SARS-CoV-2 and Epstein-Barr virus-encoded small RNA (EBER) were also performed.

Results: We observed mucosal epithelium shedding, intestinal mucosal erosion, focal inflammatory necrosis with hemorrhage, massive neutrophil infiltration, macrophage proliferation accompanied by minor lymphocyte infiltration. Fungal spores and gram positive cocci but not mycobacteria tuberculosis were identified. Immunohistochemistry staining showed abundant $\mathrm{CD}^{+} 8^{+}$macrophages but few lymphocytes infiltration. HSV, CMV and EBV were negative. ISH of SARS-CoV-2 RNA showed positive signal which mostly overlapped with CD68 positivity.

Conclusions: The in situ detection of SARS-CoV-2 RNA in intestinal macrophages implicates a possible route for gastrointestinal infection. Further study is needed to further characterize the susceptibility of enteric cells to SARS-CoV-2 infection.
\end{abstract}

Keywords: COVID-19, SARS-CoV-2, In situ hybridization, Case report

\section{Introduction}

The coronavirus disease-19 (COVID-19), caused by infection with severe acute respiratory syndrome coronavirus 2 (SARS-CoV-2) [1, 2] can cause diseases of disparate severity. As the knowledge on the clinical manifestations of COVID-19 accumulated, gastrointestinal symptoms such as diarrhea, nausea

\footnotetext{
* Correspondence: fengyanling@shphc.org.cn; luhongzhou@shphc.org.cn ${ }^{\dagger}$ Yanling Feng, Dong Zeng and Lvyin Hu contributed equally to this work. 'Department of Pathology, Shanghai Public Health Clinical center, Fudan University, Shanghai, China

${ }^{3}$ Department of Infectious Disease, Shanghai Public Health Clinical center, Fudan University, Shanghai, China

Full list of author information is available at the end of the article
}

and vomiting gradually received attention [3]. The viral RNA was found to sustain longer in fecal samples than in respiratory samples [4]. Furthermore, with the expression of ACE2 (angiotensin converting enzyme 2), enterocytes were reported to support SARS-CoV-2 replication and virus shedding [5]. Nevertheless, there has been a lack of direct evidence on the existence of virus within enteric cells. Here we report histological and molecular pathology findings based on the enteric detachment tissues from a COVID-19 patient.

(c) The Author(s). 2021 Open Access This article is licensed under a Creative Commons Attribution 4.0 International License, which permits use, sharing, adaptation, distribution and reproduction in any medium or format, as long as you give appropriate credit to the original author(s) and the source, provide a link to the Creative Commons licence, and indicate if changes were made. The images or other third party material in this article are included in the article's Creative Commons licence, unless indicated otherwise in a credit line to the material. If material is not included in the article's Creative Commons licence and your intended use is not permitted by statutory regulation or exceeds the permitted use, you will need to obtain permission directly from the copyright holder. To view a copy of this licence, visit http://creativecommons.org/licenses/by/4.0/ The Creative Commons Public Domain Dedication waiver (http://creativecommons.org/publicdomain/zero/1.0/) applies to the data made available in this article, unless otherwise stated in a credit line to the data. 


\section{Material and methods Histopathology}

Intestinal tissues were fixed with $4 \%$ neutral formaldehyde for 6-10 h, routinely dehydrated and embedded with paraffin, $4 \mu \mathrm{M}$ sections were serially cut on APES (3-Aminopropyltriethoxysilane) coated slides and stained with hematoxylin and eosin (H\&E) and observed on a light microscope. PAS (Periodic acid-Schiff) staining, Gram staining, Ziehl-Neelsen stain and Grocott's Methenamine Silver (GMS) Staining were performed using commercial kits from Baso (Zhuhai, China) according to the manufacturer's instructions.

\section{In situ hybridization}

The ViewRNA ISH Tissue Assay kit (Affymetrix, CA. USA) was used to detect the plus strand of SARS-CoV-2 RNA with probe set targeting positive strand (target region: nt705-1676, nt4356-5607, nt12808-13,943, nt20571-21,759, nt26645-27,763, nt28212-29,152, Reference LC521925.1) of the viral genome (catalog No. VPNKRHH, Thermo Fisher). The hybridization and amplification procedures were performed according to the protocols provided by the manufacturer with minor modifications. After pretreatment, hybridization and amplification, sections were finally stained with NBT and BCIP (Roche) in developing solution at $37^{\circ} \mathrm{C}$ and counterstained with nuclear fast red (Vector Labs, USA). Slides were dehydrated and mounted with Ultra-Clear (Baso, China). In situ detection of EBER was performed using a diagnostic kit from ZSGB-Bio (Beijing, China).

\section{Immunohistochemistry}

Immunohistochemistry staining was performed with Leica Bond-Max autostainer. Sections were routinely dewaxed and rehydrated. After appropriate heat induced antigen retrieval with BOND Epitope Retrival Solution1 or 2, sections were incubated with the primary monoclonal antibody against the CD68 (clone PGM, ZSBio, China), CD3 and CD4, CMV antigen and HSV antigen (Abcam) respectively. Signal was developed with 3,3'- diaminobezdine (DAB). Sections were counterstained with Mayer's hematoxylin.

\section{Detection for SARS-CoV-2 nucleic acids}

qRT-PCR of SARS-CoV-2 RNA was performed using a commercial kit (DaAn Gene, Guangzhou, China) which targets two regions (orf1a/b and $\mathrm{N}$ ) for amplification.

\section{Results}

We present a case of a man (Chinese) aged 75 who was admitted into Shanghai Public Health Clinical Center. On arriving at Shanghai from United States by airflight on March 19th 2020, he was identified to have abnormal body temperature $\left(37.7^{\circ} \mathrm{C}\right)$ and was later tested positive for SARSCoV-2 RNA. His wife, accompanying him on the flight, was diagnosed with COVID-19 1 day later. The patient had high blood pressure for 21 years and diabetes for 5 years. Computed tomography $(\mathrm{CT})$ scan indicated interstitial changes in the lower lobe of right lung. The key laboratory results during his hospitalizations were summarized in Table 1. Blood test results upon admission showed $6.0 \times 10^{9} / \mathrm{L}$ leukocyte, $1.6 \times 10^{9} / \mathrm{L}$ lymphocyte, $\mathrm{C}$ reactive protein $<5 \mathrm{mg} / \mathrm{L}$ (Table 1 ). qRT-PCR of SARS-CoV-2 RNA from the patient's throat swab showed positive result. He was treated with inhaled IFN- $\alpha$ and hydroxychloroquine. The patient's condition deteriorated on the 7th day after admission with body temperature of $39^{\circ} \mathrm{C}$ and acute respiratory distress. The gastrointestinal symptoms included abdominal pain, abdominal distension and diarrhea but no nausea or vomiting. He was soon intubated and later treated with ECMO (Extracorporeal Membrane Oxygenation). Linezolid (0.6 g q12h) was administered by nasal feeding and Tazobactam and Piperacillin was administered intravenously. Oval-shaped fungi were identified in sputum samples 15 days after admission. On day 27 , anal drainage revealed yellow paste-like feces. On day 30, the patient had loose stool of $1700 \mathrm{ml}$, accompanied by blood mucus. White spots were later found in his oral cavity which was treated with flucanazol capsule. Fecal occult blood was identified on day 33 post admission. Enteroscopic examination was performed to evaluate the degree of intestinal bleeding, which reported intestinal and colonic erosion, ulcer,

Table 1 Basic clinical parameters during hospitalization

\begin{tabular}{|c|c|c|c|c|c|c|c|}
\hline Clinical Variables & Day 2 & Day 11 & Day 27 & Day 42 & Day 45 & Day 54 & Day 71 \\
\hline Leukocyte $\left(10^{9} / \mathrm{L}\right)$ & 6.0 & 15.64 & 6.62 & 12.66 & 15.07 & 12.25 & 9.33 \\
\hline Lymphocyte $\left(10^{9} / \mathrm{L}\right)$ & 1.6 & 0.63 & 0.36 & 0.76 & 0.44 & 0.85 & 0.50 \\
\hline CD3 T lymphocyte (/ul) & 929 & 239 & 280 & 581 & 240 & 719 & 467 \\
\hline Neutrophil $\left(10^{9} / \mathrm{L}\right)$ & 4.5 & 14.58 & 5.15 & 9.76 & 14.63 & 10.41 & 8.51 \\
\hline SARS-CoV-2 RNA (-/+) & + & + & + & + & + & + & - \\
\hline$C$ reactive protein $(\mathrm{mg} / \mathrm{L})$ & $<5$ & 111.32 & 127.75 & 50.21 & 117.39 & 29.41 & 28.36 \\
\hline IL-6 (pg/ml) & 0 & 387.82 & 11.26 & 19.64 & 1128.07 & 46.96 & 15.91 \\
\hline IL-8 (pg/ml) & 0.07 & 52.54 & 22.95 & 21.34 & 34.03 & 22.88 & 30.25 \\
\hline
\end{tabular}



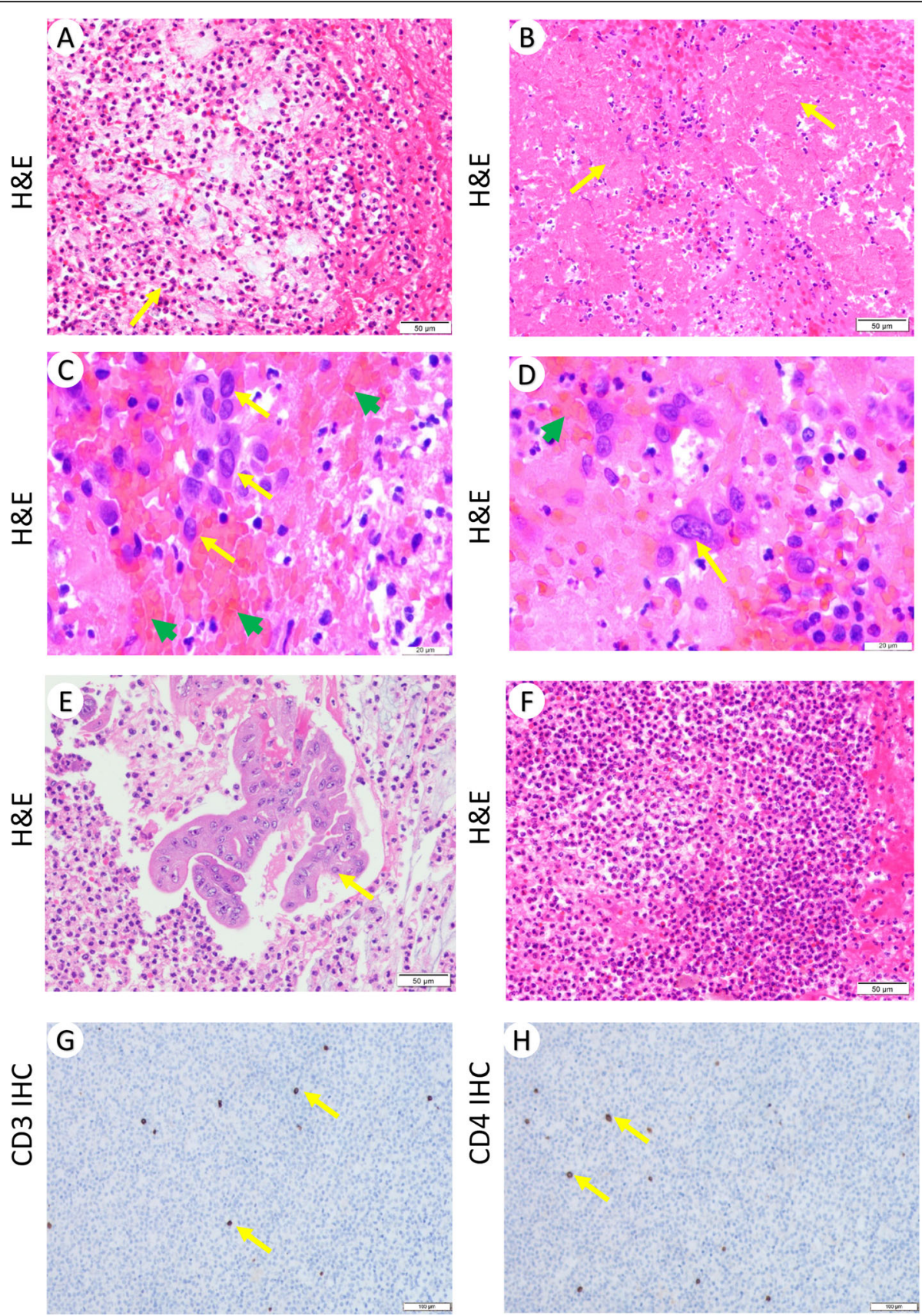

Fig. 1 Histopathological findings in the intestinal tissues. a Massive neutrophil infiltration accompanied by fibrin, erythrocyte and mucus exudation (200X). b focal inflammatory necrosis (200X); c-d enlarged nuclei and deep staining of macrophages (yellow arrow) with prominent nucleoli, focal hemorrhage accompanied by neutrophil and lymphocyte infiltration (400X). e residual glandular epithelial cells with hemorrhage necrosis, fibrin and mucus exudation (200X). f Focal purulent inflammation (200X). g Immunohistochemistry of CD3 (100X), $\mathbf{h}$

Immunohistochemistry of CD4 (100X)

hyperplasia, bleeding and mucus exudation. Hemostatic drug flushing was performed during enteroscopy. On day 45, the blood test showed $15.07 \times 10^{9} / \mathrm{L}$ leukocyte, highly abundant neutrophil (97.10\% in leukocyte), low lymphocyte counts $\left(0.44 \times 10^{9} / \mathrm{L}\right)$, low $\mathrm{CD} 3 \mathrm{~T}$ cell count $(240 / \mu \mathrm{l}), \mathrm{C}$ reactive protein $117.39 \mathrm{mg} / \mathrm{L}$ and prothrombin time $27.50 \mathrm{~s}$.
Extremely high level of inflammatory cytokines such as IL$61128.07 \mathrm{pg} / \mathrm{ml}$ and IL-8 $34.03 \mathrm{pg} / \mathrm{ml}$ were reported (Table 1). The patient's throat swab, feces and bronchial lavage fluid were all tested positive for SARS-CoV-2. CT scans showed bilateral scattered patchy high-density regions and pleural effusion. After intensive care and treatment, the patient finally 
recovered 60 days after hospitalization and viral RNA was negative.

The mucous and necrotic tissues in feces were collected on day 47 and 49 after admission for pathology. Three blocks of pale and beige enteric tissues with sizes of $3 \times 2 \times 1 \mathrm{~cm}, 4 \times 2 \times 1.5 \mathrm{~cm}, 2.5 \times 1.5 \times 0.8 \mathrm{~cm}$ were examined. Under the microscope, we observed massive neutrophil infiltration (Fig. 1a, arrow), focal inflammatory necrosis (Fig. 1b, arrow) and hemorrhage (Fig. 1c-d, green arrow), mucosal epithelium shedding and erosion (Fig. 1e, arrow), accompanied by abscess (Fig. 1f). Macrophage infiltration (Fig. 1c-d, yellow arrow) and few lymphocytes (Fig. 1g-h, arrow) were found. To examine the possible microbiological infection, PAS staining and GMS Staining as well as Gram staining were performed. Significant number of round and ovalshape fungal spores (Fig. 2a-b, arrow) but not pseudohypha were observed. Mass spectrometry of isolated
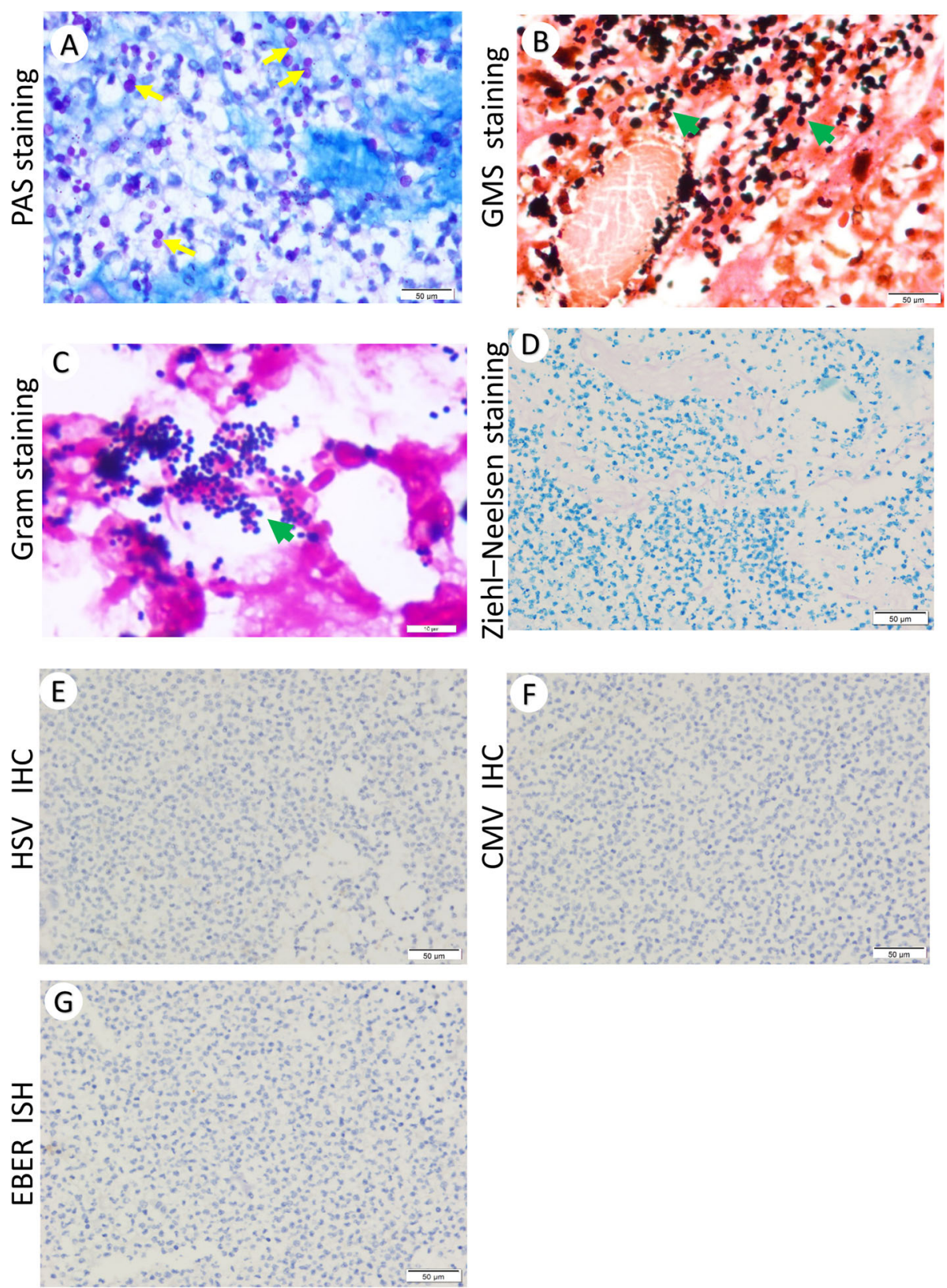

Fig. 2 Possible microbiological infections in the intestinal tissue. a Fungal spores (purple red, yellow arrows) but without pseudohypha were identified by PAS staining (400X), b Fungal spores (black, green arrows) were identified by GMS staining (400X), c Gram staining revealed positive cocci (purple, green arrow, 1000X). d Ziehl-Neelsen stain (400X) did not identified Mycobacterium tuberculosis. Immunohistochemistry of HSV (e 400X) and CMV (f 400X) and in situ hybridization of EBV (g 400X) did not showed signal 
colonies identified them to be Candida galbrata. Gram positive cocci (Fig. 2c, arrow) were also found. No mycobacteria were identified using Ziehl-Neelsen stain (Fig. 2d). Immunohistochemistry for HSV (Fig. 2e) and CMV antigens (Fig. 2f) were negative. In situ hybridization of EBER RNA was negative (Fig. 2g).

Next, we performed in situ hybridization of SARSCoV-2 RNA. The blue purple signal was observed in the cytoplasm (Fig. 3a-b, arrow). The specificity of the in situ assay was confirmed by the lack of background in enteric tissues collected from uninfected patients (Fig. 3e). Immunostaining of the adjacent sections of the same tissues showed a large number of $\mathrm{CD} 68^{+}$positive macrophages (Fig. 3c-d). On the other hand, CK20 positive (epithelial) cells were very rare (Fig. 3f, arrow) in these tissues. No viral RNA positive cells were found to reside in these cells.

\section{Discussion}

Although SARS-CoV-2 shares around $80 \%$ sequence similarity with SARS-CoV, its clinical manifestations are far more variable, ranging from asymptomatic carrier to life-threatening respiratory failure. The risk factors associated with disease severity were host factors such as older age and comorbidities [6]. Apart from pulmonary disease, diarrhea was consistently documented as a minor manifestation of COVID-19. Importantly, the percentage of patients showing GI symptoms increases during hospitalization [7]. In this reported case, the pathological findings indicated excessive inflammatory responses such as neutrophil infiltration, macrophage hyperplasia which resulted in excessive intestinal damages such as mucosal epithelium shedding erosion and focal inflammatory necrosis. Additional staining revealed the colonization of fungi and pathogenic Gram-positive bacteria. Indeed, severe SARS-CoV-2 infection can cause systemic
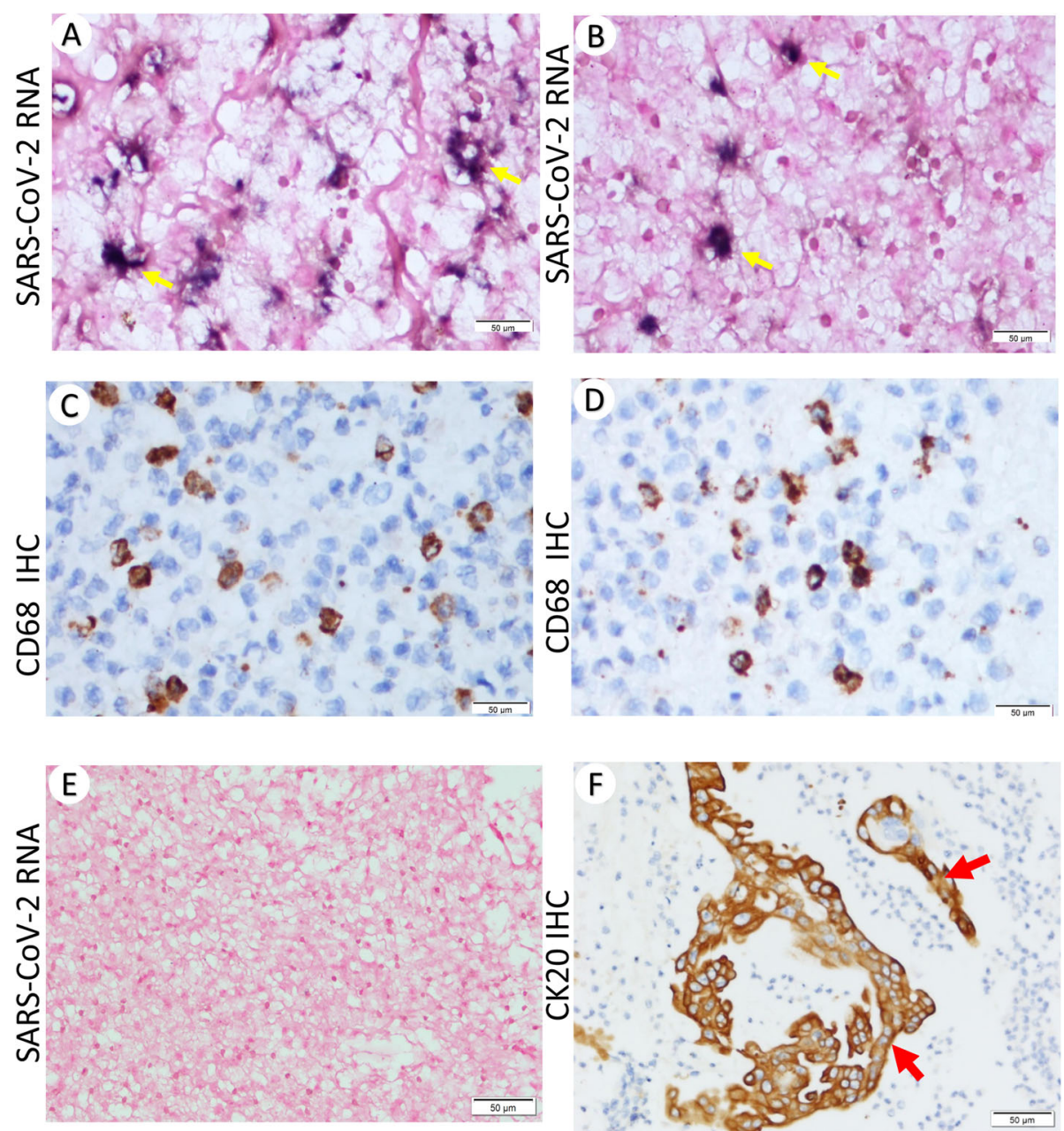

Fig. 3 In situ detection of SARS-COV-2 RNA in intestinal macrophages. $\mathbf{a}$, b In situ hybridization of SARS-CoV-2 RNA (400X) c, d Immunohistochemistry of CD68 (400X). e in situ hybridization of SARS-CoV-2 RNA in enteric tissue of uninfected individual, $\mathbf{f}$ Immunohistochemistry of CK20 (400X). $\mathbf{a}$ and $\mathbf{c}, \mathbf{b}$ and $\mathbf{d}$ are from same regions of adjacent sections 
inflammatory response which could significantly disturb the homeostasis of the gut microbiota $[8,9]$ and facilitate the growth of pathogenic microbes. In the case reported here, GI symptom was observed on day 27 after admission and coincided with fungal and bacterial infection in the intestine. The older age and co-existing conditions may further deteriorate the intestinal micro-environment.

Apart from the impact of altered intestinal microflora, SARS-CoV-2 may play a direct role in the gastrointestinal symptoms. Transcriptomic and proteomic profiling in various human tissues indicated that intestinal tissue expresses the most abundant ACE2 [10]. Indeed, productive infection was reported in primary human enteroid culture [5]. Three potential mechanisms were thought to explain the spread of SARS-CoV-2 into the gut. 1), The viral particles released into circulation could spread to other susceptible organs; 2), SARS-CoV-2 could be consistently detected in posterior oropharyngeal saliva most probably shed by upper respiratory cells [11] which may be responsible for a large part of viral input into the GI tract and hence enterocyte infection; 3), Alveolar macrophage, which also expresses ACE2, could be a carrier of this virus [12] and facilitate extra-pulmonary infection via macrophage trafficking. Indeed, Wang et al. reported the positive staining of viral nucleocapsid protein in not only pneumocytes but also alveolar macrophages [12]. In the case reported here, the viral RNA signal was found to reside in tissues with abundant infiltrated macrophages which might support the third hypothesis. Nevertheless, due to the very few intestinal epithelial cells detected in the tissues, the possibility of enterocyte infection in vivo cannot be conclusively ruled out.

Taken together, we reported comprehensive pathological analysis of one COVID-19 case with severe secondary gastrointestinal disease. These serious manifestations were the combined result of a series of microbiological insults, e.g., bacterial and fungal colonization, as a result of prolonged respiratory failure in addition to enteric viral infection. The results of molecular pathology analysis suggested the role of macrophage in spreading SARS-CoV-2 into the gastrointestinal tract. Additional study is needed to clarify the in vivo viral susceptibility profile of intestinal cell types.

\begin{abstract}
Abbreviations
COVID-19: Coronavirus disease-19; ISH: In situ hybridization; SARS-CoV-2: Severe acute respiratory syndrome coronavirus 2; CMV: Cytomegalovirus; HSV: Herpes Simplex Virus; EBER: Epstein-Barr virus-encoded small RNA; GMS: Grocott's Methenamine Silver; ACE2: Angiotensin converting enzyme 2; CT: Computed tomography; Gl: Gastrointestinal; CK7: Cytokeratin 7; CK20: Cytokeratin 20
\end{abstract}

\section{Authors' contributions}

YF, HL conceived the study. DZ, LH, YY, SS, YS, JX, WG performed the experiments. YL, TQ, QW, FL, LC collected the samples. YF, HL analyzed the data. DZ, LH drafted the manuscript, YF and HL reviewed and approved the manuscript. The author(s) read and approved the final manuscript.

\section{Funding}

This work is supported by National Megaprojects of China for Infectious Diseases (2017ZX10103009-001, 2018ZX10305409-001-005). It is also funded by Shanghai Science and Technology Commission (20431900103); Fudan University (IDF162005); Zhejiang University (2020XGZX030) Shanghai Public Health Clinical Center (2020YJKY01) and Shanghai Major Projects on Infectious Diseases (shslczdzk01102).

\section{Availability of data and materials}

The relevant data and materials pertaining to this study are available upon request.

\section{Declarations}

\section{Ethics approval and consent to participate}

This study was approved by the ethics committee of the Shanghai Public Health Clinical Center (Approval No. YJ-2020-S015-01). Informed consent was obtained from the patient's family member.

\section{Competing interests}

The authors declare that no conflict of interests exists.

\section{Author details}

${ }^{1}$ Department of Pathology, Shanghai Public Health Clinical center, Fudan University, Shanghai, China. ${ }^{2}$ Department of Clinical Laboratory, Shanghai Public Health Clinical center, Fudan University, Shanghai, China. ${ }^{3}$ Department of Infectious Disease, Shanghai Public Health Clinical center, Fudan University, Shanghai, China. ${ }^{4}$ Department of Respiratory Medicine, Shanghai Public Health Clinical center, Fudan University, Shanghai, China. ${ }^{5}$ Department of Digestive Diseases, Shanghai Public Health Clinical center, Fudan University, Shanghai, China.

Received: 27 August 2020 Accepted: 3 March 2021

Published online: 05 May 2021

\section{References}

1. Zhou P, et al. A pneumonia outbreak associated with a new coronavirus of probable bat origin. Nature. 2020;579(7798):270-3.

2. Zhu N, et al. A novel coronavirus from patients with pneumonia in China, 2019. N Engl J Med. 2020;382(8):727-33.

3. Guan WJ, et al. Clinical characteristics of coronavirus disease 2019 in China. N Engl J Med. 2020;382(18):1708-20. https://doi.org/10.1056/NEJMoa2002032.

4. Zhang W, et al. Molecular and serological investigation of 2019-nCoV infected patients: implication of multiple shedding routes. Emerg Microbes Infect. 2020;9(1):386-9.

5. Zang R, et al. TMPRSS2 and TMPRSS4 promote SARS-CoV-2 infection of human small intestinal enterocytes. Sci Immunol. 2020;5(47):eabc3582.

6. Zhang $X$, et al. Viral and host factors related to the clinical outcome of COVID-19. Nature. 2020;583(7816):437-40.

7. Song $Y$, et al. SARS-CoV-2 induced diarrhoea as onset symptom in patient with COVID-19. Gut. 2020;69(6):1143-4.

8. Zuo T, et al. Alterations in gut microbiota of patients with COVID-19 during time of hospitalization. Gastroenterology. 2020;159(3):944-955.e8.

9. Gu S, et al. Alterations of the gut microbiota in patients with COVID-19 or H1N1 influenza. Clin Infect Dis. 2020:71(10):2669-78.

10. Hikmet $F$, et al. The protein expression profile of ACE2 in human tissues. bioRxiv. 2020;03(31):016048.

11. To, K.K, et al. Temporal profiles of viral load in posterior oropharyngeal saliva samples and serum antibody responses during infection by SARS-CoV-2: an observational cohort study. Lancet Infect Dis. 2020:20(5):565-74.

12. Wang $C$, et al. Alveolar macrophage dysfunction and cytokine storm in the pathogenesis of two severe COVID-19 patients. EBioMedicine. 2020;57:102833.

\section{Publisher's Note}

Springer Nature remains neutral with regard to jurisdictional claims in published maps and institutional affiliations. 